\section{Demenz und B-Vitaminmangel: Worin besteht der Zusammenhang?}

Ein Schwerpunkt des diesjährigen Kongresses 7th International Conference on Homocysteine Metabolism, 21.-25. Juni 2009 in Prag, war die Primärprävention von Demenz. Homocystein gilt derzeit als der stärkste Prädiktor für kognitiven Abbau und Hirnatrophie. Erst etwa 27 Jahre nach Auftreten der ersten durch $\mathrm{M}$. Alzheimer verursachten Läsionen im Hippocampus kommt es zu kognitiven Störungen, die sich im Verlauf von weiteren 5 Jahren zu einer Demenz entwickeln [1]. Unter den verfügbaren Parametern ist Homocystein der stärkste Prädiktor für kognitiven Abbau und Hirnatrophie [2-3]. Im Oxford Healthy Aging Project (OHAP) zur Kognition im Alter hat sich ge-
Forsch Komplementmed 16 | 6 | 09

zeigt, dass der kognitive Abbau bei Personen mit Homocys-teinspiegeln über $20 \mu \mathrm{mol} / \mathrm{L}$ um $50 \%$ stärker war als bei jenen mit $10 \mu \mathrm{mol} / \mathrm{L}$ [4]. In 2008 hat eine Studie des Oxford Project To Investigate Memory and Aging (OPTIMA) ergeben, dass ein schlechter VitaminB12-Status mit einem Verlust an Hirnvolumen im MRT assoziiert ist [5]. Eine aktuelle Studie aus Schweden zur Entwicklung von Risikofaktoren bei 1368 mittelalten Frauen, die über 35 Jahre lang beobachtet wurden, ergab, dass nur Homocystein mit einem erhöhten Risiko für M. Alzheimer assoziiert war - andere Risikofaktoren hielten der statistischen Analyse nicht stand [6].

Homocystein ist ein Labormarker für einen Mangel an Folsäure, Vitamin B12 oder B6 durch schlechte Ernährung, Resorptionsstörungen oder Medikamenteninteraktionen und aus neurobiologischer Sicht ein prognosti- scher Parameter für das Risiko kognitiver Beeinträchtigung und Demenz, eine beschleunigte Gehirnatrophie, und einen schnelleren kognitiven Abbau bei manifester Alzheimer Demenz.

\section{Quellen}

1 Smith A D.: Proc Natl Acad Sci U S A 2002;99(7): 4135-7.

2 Ravaglia G et al: Am J Clin Nutr 2005:82(3):636-43.

3 Oulhaj A et al.: Int J Geriatr Psychiatry 2009, [Epub ahead of print].

4 Clarke R et al.: Am J Clin Nutr 2007;86(5):1384-91.

5 Vogiatzoglou A et al: Neurology 2008;71(11):826-32.

6 Zylberstein DE et al.: Neurobiol Aging 2009 [Epub ahead of print].

Weitere Informationen bei

Patzer PR GmbH / Dr. Maren Mundt

Steinheilstraße 10, 85737 Ismaning

Tel. +4989 5527939-49

maren.mundt@patzer-pr.com

\section{Silibinin: Antivirale Wirkung bei Hepatitis-C-Patienten}

Aufgrund der schwerwiegenden Langzeitfolgen benötigen Patienten mit einer chronischen Hepatitis-C-Infektion (HCV) so schnell wie möglich eine wirksame Therapie. Neuen aussichtsreichen Daten zufolge verfügt Silibinin über deutliche antivirale Effekte bei chronischen HCV-Infektionen, die nicht auf die Standardtherapie mit pegyliertem Interferon in Kombination mit Ribavirin ansprechen. Vor Kurzem präsentierten die Mediziner Dr. Michael Biermer und Professor Thomas Berg beeindruckende Ergebnisse aus ersten Therapieversuchen mit Silibinin an der Charité in Berlin [1]. Die Erkenntnisse von Biermer und Berg bestätigen die im Jahr 2008 von Professor Peter Ferenci aus Wien veröffentlichten Pilotdaten über die antiviralen Eigenschaften von Silibinin [2]. Bei Silibinin handelt es sich um die Hauptkomponente von Silymarin, einem Extrakt aus der Mariendistel. Beide Arbeitsgruppen verwendeten intravenös verabreichtes Silibinin (Legalon ${ }^{\circledR}$ SIL) - ein hochwirksames und gut verträgliches Arzneimittel, bisher zugelassen als Antidot bei einer Vergiftung mit Knollenblätterpilzen.

Erste Hinweise über einen möglichen Wirkmechanismus lieferten In-vitro-Experimente, die zeigten, dass Silibinin die für die HCVReplikation notwendige Polymerase signifikant hemmt [3]. Die vorliegenden Daten weisen darauf hin, dass Silibinin (Legalon ${ }^{\circledR}$ SIL) für Patienten mit einer chronischen HCV-Infektion, die auf die Standardtherapie nicht ansprechen, eine effektive Therapieoption darstellt.
Die Fachwelt setzt jetzt auf Ergebnisse weiterer Studien.

\section{Quellen}

1 Vortrag von Dr. Michael Biermer und Professor Thomas Berg. Antivirale Therapie mit Silibinin bei Patienten mit chronischer Interferon/Ribavirin refraktärer Hepatitis C. 7. Berliner Kongress Phytotherapie 2009, 10.-12. September, Berlin.

2 Ferenci P et al.: Gastroenterology 135;5:1561-1567.

3 Präsentation Prof. Peter Ferenci, im Rahmen des Kongresses der European Association for the Study of the Liver (EASL), Kopenhagen, 2009.

Weitere Informationen bei

Adlexis GmbH

Karin Bretz

Liebherrstr. 10, 80538 München

Tel. +49 89 2020816-0, Fax -10

karin.bretz@adlexis.com

\title{
Ticker+++ Ticker+++ Ticker+++ Ticker+++ Ticker+++ Ticker+++ Ticker+++
}

Cefak KG. Neue Erkenntnisse und Studien in der Forschung zeigen Zusammenhänge von Selen, Schilddrüse und Schwangerschaft auf, ebenso wie den Stellenwert einer guten Selenversorung bei Blasenkrebs. Neben einem ausführlichen Therapieteil finden sich in der erweiterten 3. Auflage des «Selen-Manuals» Angaben zu den Grundlagen einer Selen-Therapie sowie ein Patienten-Service. Das «Selen-Manual» kann kostenlos bestellt werden bei:

Cefak KG

Cefasel-Service

cefak@cefak.com

www.selen-info.de
Dr. Loges \& Co. GmbH. Eine neue Wirkstoffkombination mit natürlichen Substanzen zur Senkung erhöhter Lipidwerte sorgt in der naturheilkundlichen Fachwelt für Aufsehen. Neueste Studien konnten zeigen, dass die Kombination aus Tocotrienolen («ungesättigtes Vitamin E») aus Palmölfrüchten sowie speziellen Flavonoiden aus Citrusfruchtschalen effektiv Blutlipide senken kann. Das Präparat lipoCorrect ${ }^{\circledR}$ ist rezeptfrei in der Apotheke erhältlich.

Weitere Informationen bei ifemedi Institut für ernährungsmedizinische Information presse@medizin-ernaehrung.de
Heel GmbH. Interessierte Ärzte, Apotheker, Heilpraktiker und Tierärzte können ab sofort umfassende Informationen rund um die Medizin der Biologischen Heilmittel Heel im Fachbereich der neu gestalteten Internetpräsenz www.heel.de abrufen. Mit dem neu konzipierten Internetauftritt soll künftig noch mehr Wissen über moderne Behandlungsmethoden mit homöopathischen Kombinationsarzneimitteln abrufbar sein.

Weitere Informationen bei

Schuster Public Relations \& Media

Consulting $\mathrm{GmbH}$

Verena Zinn

zinn@schuster-pr.de 